\title{
Möbius Fermions: Improved Domain Wall Chiral Fermions *
}

\author{
Richard C. Brower ${ }^{\mathrm{a}}$, Hartmut Neffa and Kostas Orginos ${ }^{\mathrm{b}}$

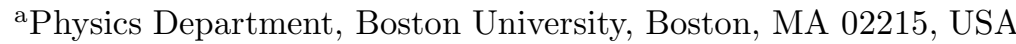 \\ ${ }^{\mathrm{b}}$ Center for Theoretical Physics, MIT, Cambridge, MA 02139, USA
}

A new class of domain wall fermions is defined that interpolates between Shamir's and Boriçi's form without increasing the number of Dirac applications per CG iteration. This class represents a full (real) Möbius transformation of the Wilson kernel. Simulations on quenched Wilson lattices with $\beta=6.0$ show that the number of lattice sites $\left(L_{s}\right)$ in the fifth dimension can be reduced by a factor of 2 or more at fixed value of chiral symmetry violations measured by the residual mass $\left(m_{\text {res }}\right)$.

\section{INTRODUCTION}

Chiral fermions based on the Ginsparg-Wilson relations give a rigorous approach to exact chiral symmetry at finite lattice spacing. However this clear theoretical advance requires efficient algorithms to allow them to be used routinely in lattice gauge theory simulations. The two major implementations base on the overlap form of Neuberger [1] or the domain wall of Kaplan [2, adapted by Shamir [3] and Boriçi [4 can both be seen as a means to satisfy the zero quark mass Ginsparg-Wilson relation,

$\gamma_{5} D_{o v}(0)+D_{o v}(0) \gamma_{5}-2 D_{o v}(0) \gamma_{5} D_{o v}(0)=2 \gamma_{5} \Delta_{L_{s}}$

where the error $\Delta_{L_{s}} \rightarrow 0$ is made as small as needed by taking $L_{s} \rightarrow \infty$, where $L_{s}$ parameterizes the order of a rational polynomial approximation. A general solution, including a non-zero quark mass $\mathrm{m}$, is provided by the overlap operator,

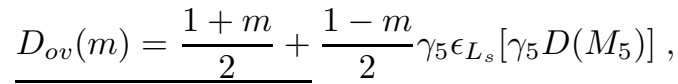

*This is supported in part by Department of Energy under Contracts No. DE-FG0291ER40676 and DFFC0294ER40818 where $\epsilon_{L_{s}}[x]$ is the rational approximation of the sign function, $\epsilon[x]=x /|x|$ and $\Delta_{L_{s}}[x] \equiv(1-$ $\left.\epsilon_{L_{s}}^{2}[x]\right) / 4$. In fact the GW relation defines an infinite class of chiral fermions depending on the selection of the kernel $D\left(M_{5}\right)$, the simplest but certainly not the ideal example being the Wilson fermion operator itself,

$$
\begin{aligned}
& D_{w}\left(M_{5}\right)=\left(4+M_{5}\right) \delta_{x, y}- \\
& \frac{1}{2}\left[\left(1-\gamma_{\mu}\right) U_{\mu}(x) \delta_{x+\mu, y}+\left(1+\gamma_{\mu}\right) U_{\mu}^{\dagger}(y) \delta_{x, y+\mu}\right],
\end{aligned}
$$

with mass $M_{5}=O(-1)$. On the other hand the standard Shamir implementation of Domain Wall fermions is equivalent to an overlap kernel,

$D_{\text {Shamir }}\left(M_{5}\right)=\frac{a_{5} D_{w}\left(M_{5}\right)}{2+a_{5} D_{w}\left(M_{5}\right)}$.

Here we suggest a modification of the standard Shamir domain wall approach that replaces the overlap kernel, or 5-th time Hamiltonian $\gamma_{5} D\left(M_{5}\right)$, by a real Möbius transformation of the Wilson operator: $D_{w}\left(M_{5}\right) \rightarrow\left(a+b D_{w}(-1) /(c+\right.$ $\left.d D_{w}(-1)\right)$ or equivalently in a more convenient parameterization for the domain wall formulation,

$D_{\text {Mobius }}\left(M_{5}\right)=\frac{\left(b_{5}+c_{5}\right) D_{w}\left(M_{5}\right)}{2+\left(b_{5}-c_{5}\right) D_{w}\left(M_{5}\right)}$. 
In addition to the Shamir parameter, $a_{5}=b_{5}-c_{5}$, there is a new independent scaling factor, $\alpha=$ $c_{5}+b_{5}$, which turns out to be a significant advantage at finite $L_{s}$ even though it is irrelevant at $L_{s}=\infty$ due scale invariance of the sign function: $\epsilon[\alpha x]=\epsilon[x]$. One way to understand the advantage of the Möbius transform is to notice that it can map any 3 real values (e.g. eigenvalues of $D_{w}$ ) to arbitrary points. This allows one to choose $M_{5}$ to separate legitimate chiral modes from doublers and simultaneously to adjust both the smallest and largest real eigenvalue in $D\left(M_{5}\right)$. Note also Möbius fermions have a continuous path to conventional overlap kernel as implemented by Boriçi's with $b_{5}=c_{5}=a_{5}$.

\section{DOMAIN WALL IMPLEMENTATION}

It is very natural to implement the real Möbius kernel by the 5 -d Domain Wall matrix, $D_{D W}(m)$ :

$$
\left[\begin{array}{cccc}
D_{+}^{(1)} & D_{-}^{(1)} P_{-} & \cdots & -m D_{-}^{(1)} P_{+} \\
D_{-}^{(2)} P_{+} & D_{+}^{(2)} & \cdots & 0 \\
\cdots & \cdots & \cdots & \cdots \\
-m D_{-}^{\left(L_{s}\right)} P_{-} & 0 & \cdots & D_{+}^{\left(L_{s}\right)}
\end{array}\right]
$$

where $D_{+}^{(i)}=b_{5} \omega_{i} D_{w}\left(M_{5}\right)+1$ and $D_{-}^{(i)}=$ ${ }_{c} \omega_{i} D_{w}\left(M_{5}\right)-1$. In this discussion we simplify to the case of polar decomposition $\left(\omega_{i}=1\right)$, although Zolotarev polynomials $\left(\omega_{i} \neq 1\right)$ can be implemented as well. By standard LDU decomposition [5] for $D_{D W}(m)$, this leads back to an effective overlap operator, with

$\epsilon_{L_{s}}[H]=\frac{(1+H)^{L_{s} / 2}-(1-H)^{-L_{s} / 2}}{(1+H)^{L_{s} / 2}+(1-H)^{-L_{s} / 2}}$

given by the polar decomposition for the "Hamiltonian" $H=\gamma_{5} D_{\text {Mobius }}\left(M_{5}\right)$ defined above. The detailed algebraic steps will be published in a forthcoming article. The chiral modes at the boundary

$q_{x}=P_{-} \Psi_{x, 1}+P_{+} \Psi_{x, L_{s}}$
$\bar{q}_{y}=-\left[\bar{\Psi}_{1} D_{-}^{(1)}\right]_{y} P_{+}-\left[\bar{\Psi}_{L_{s}} D_{-}^{\left(L_{s}\right)}\right]_{y} P_{-}$

give the direct connection to the overlap propagator,

$\left\langle q_{x} \bar{q}_{y}\right\rangle=\frac{1}{1-m}\left[D_{o v}^{-1}(m)-1\right]_{x y}$.

A key observation for this action (as well as Boriçi's and Chiu's examples) is that the new version of "gamma 5" Hermiticity for the Möbius domain wall requires pulling out a factor $D_{-}=$ $\operatorname{Diag}\left[D_{-}^{(1)}, D_{-}^{(2)}, \cdots, D_{-}^{\left(L_{s}\right)}\right]$ as well as reflecting, $\mathcal{R}$, in the fifth dimensions:

$$
\gamma_{5} \mathcal{R} D_{-}^{-1} D_{D W}(m)=\left(D_{-}^{-1} D_{D W}(m)\right)^{\dagger} \gamma_{5} \mathcal{R}
$$

This in turn is reflected in the proper definition of chiral boundary states.

\section{DEFINITION OF RESIDUAL MASS}

The chiral breaking operator $\Delta_{L_{s}}(x)$ defined above provides the breaking term in the 4 -d Noether theorem,

$\delta\left(\bar{\psi} D_{o v}(m) \psi\right)=m \bar{\psi}\left(\gamma_{5}+\widehat{\gamma}_{5}\right) \psi+2(1-m) \bar{\psi} \gamma_{5} \Delta_{L_{s}} \psi$.

Therefore its matrix elements are the proper measure of the approach to exact chirality for $L_{s} \rightarrow$ $\infty$. Physically relevant matrix elements should be sensitive to long distance (IR) physics. In the Domain Wall language, there is a corresponding breaking term in axial Ward-Takahashi identity,

$$
\Delta_{\mu} J_{\mu}^{a, D W}(x)=2 m \bar{q}_{x} \lambda^{a} \gamma_{5} q_{x}+2 \bar{Q}_{x} \gamma_{5} \lambda^{a} Q_{x}
$$

where the $Q_{x}, \bar{Q}_{x}$ fields lie on any plane separating the left and right domain walls. A convenient measure of the breaking term (or residual mass) is given by

$$
m_{r e s}(t) \equiv \frac{\sum_{\vec{x}}\left\langle\bar{Q}_{\vec{x}, t} \gamma_{5} Q_{\vec{x}, t} \bar{q}_{0} \gamma_{5} q_{0}\right\rangle_{c}}{(1-m)^{2} \sum_{\vec{x}}\left\langle\bar{q}_{\vec{x}, t} \gamma_{5} q_{\vec{x}, t} \bar{q}_{0} \gamma_{5} q_{0}\right\rangle_{c}}
$$

in the plateau region with $t$ away from the sources. An alternative definition (equivalent in 
infinite volumes) is found by summing over all time slices and removing the contact term in Eq. 5. This expression for $m_{\text {res }}$ measures a specific matrix element of $\Delta_{L_{s}}$,

$m_{r e s} \equiv \frac{\operatorname{Tr}\left[\Delta_{L_{s}}(H) D_{o v}^{-1} D_{o v}^{\dagger-1}\right]}{\operatorname{Tr}\left[D_{o v}^{-1} D_{o v}^{\dagger-1}\right]}=\sum_{\lambda} \rho(\lambda) \Delta_{L_{s}}(\lambda)$

The positive definite kernel guarantees that zero residual mass implies the exact Ginsparg-Wilson relations and unbroken Ward-Takahashi relations.

\section{NUMERICAL RESULTS}

In order to have the performance of Möbius (or Boriçi) fermions on a equal footing with Shamir, one must be able to use even-odd preconditioning. However the 5-d even-odd partition is impractical because the new operator $D_{-}$connects even and odd sites so that one must invert it in each CG step. Instead we define the even-odd pattern only on each 4-d slice, not alternating in 5th axis. Now the result is that even-even and oddodd matrices are independent of the gauge fields and can be inverted analytically at negligible cost. Performance tests have shown that this new 4-d even-odd precondition results in an improvement factor of roughly 3 just as the 5 -d even-odd had done for Shamir.

We tested the chirality and convergence of the Möbius domain wall operator on 20 quenched lattices at $\beta=6.0$ from the NERSC gauge connection. Our results were compared with the standard Shamir fermions for a bare quark mass $m=0.06$, resulting in a pion mass of roughly 0.4 (in lattice units) for $L_{s}=16$. All comparisons were done for standard Shamir operator with $a_{5} \equiv b_{5}-c_{5}=1.0$. We tuned $M_{5}$ and the scale $\alpha$. The pion masses were adjusted so that $L_{s}=8$ coincided with Shamir at $L_{s}=16$. For $L_{s}=8$ our tests found that $M_{5}=-1.5$ is the op-

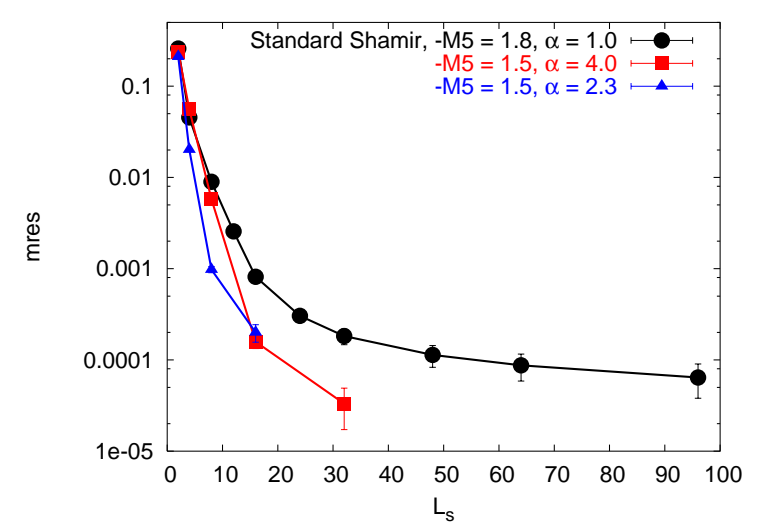

Figure 1. Comparison of $m_{r e s}$ vs $L_{s}$ for Möbius vs standard Shamir.

timal choice, which is not surprising since it sits at the mid point between the two Aoki phases at -2.2 and -0.8 . We found for $L_{s}=8$ that the optimal scale factor was $\alpha=2.3$. Furthermore we explored $m_{r e s}$ at larger $L_{s}$. There appears to be a crossover at $L_{s}=16$ for $\alpha=4$.

More detailed performance data for a variety of quench and unquenched lattices is needed, but our preliminary observations are $(1)$ at $m_{\text {res }}=$ $O(0.1 \%)$ suitable for HMC simulations, $L_{s}=8$ gives essentially the same $m_{\text {res }}$ as conventional Shamir with $L_{s}=16,(2)$ at large values of $L_{s}$ suitable for valence quarks, $m_{r e s}$ drops very rapidly if we use large scale factors and (3) finally at fixed $m_{\text {res }}$, the number of CG iterations increases only modestly for larger scale factors.

\section{REFERENCES}

1. H. Neuberger, Phys. Lett B417 (1998).

2. David B. Kaplan, Phys. Lett. B288 (1992).

3. Y. Shamir, Nucl. Phys. B406 (1993).

4. A. Boriçi, Nucl.Phys.Proc.Suppl. 83 (2000).

5. Edwards and Heller, Phys. Rev. D63 (2001). 